\title{
A topological study of contextuality and modality in quantum mechanics
}

\author{
Graciela Domenech ${ }^{* 1,3}$, Hector Freytes ${ }^{2}$ and Christian de Ronde ${ }^{3,4}$
}

March 26, 2008

\author{
1. Instituto de Astronomía y Física del Espacio (IAFE) \\ Casilla de Correo 67, Sucursal 28, 1428 Buenos Aires, Argentina \\ 2. Dipartimento di Scienze e Pedagogiche e Filosofiche - Universita degli Studi di \\ Cagliari - Via Is Mirrionis 1, 09123, Cagliari - Italia \\ 3. Center Leo Apostel (CLEA) \\ 4. Foundations of the Exact Sciences (FUND) \\ Brussels Free University - Krijgskundestraat 33, 1160 Brussels - Belgium
}

\begin{abstract}
Kochen-Specker theorem rules out the non-contextual assignment of values to physical magnitudes. Here we enrich the usual orthomodular structure of quantum mechanical propositions with modal operators. This enlargement allows to refer consistently to actual and possible properties of the system. By means of a topological argument, more precisely in terms of the existence of sections of sheaves, we give an extended version of Kochen-Specker theorem over this new structure. This allows us to prove that contextuality remains a central feature even in the enriched propositional system.
\end{abstract}

Keywords: contextuality, sheaves, modal, quantum logic

\section{Introduction}

Modal interpretations of quantum mechanics (Dieks, 1988; Dieks, 1989; Dieks, 2005; van Fraassen, 1991) face the problem of finding an objective

\footnotetext{
${ }^{*}$ Fellow of the Consejo Nacional de Investigaciones Científicas y Técnicas (CONICET)
} 
reading of the accepted mathematical formalism of the theory, a reading "in terms of properties possessed by physical systems, independently of consciousness and measurements (in the sense of human interventions)" (Dieks, 2005). These interpretations intend to consistently include the possible properties of the system in the discourse looking for a new link between the state of the system and the probabilistic character of its properties, thus sustaining that the interpretation of the quantum state must contain a modal aspect. The name modal interpretation was for the first time used by B. van Fraassen (van Fraassen, 1981) following modal logic, precisely the logic that deals with possibility and necessity. Within this frame, a physical property of a system means "a definite value of a physical quantity belonging to this system; i.e., a feature of physical reality" (Dieks, 2005). As usual, definite values of physical magnitudes correspond to yes/no propositions represented by orthogonal projection operators acting on vectors belonging to the Hilbert space of the (pure) states of the system (Jauch, 1996).

Formal studies of modal interpretations of quantum logic exist which are similar to the modal interpretation of the intuitionistic logic (Dalla Chiara, 1981; Goldblatt, 1984). A description of that those kind of approaches may be found in (Dalla Chiara et al., 2004). At first sight, it may be thought that the enrichment of the set of (actual) propositions with modal ones could allow to circumvent the contextual character of quantum mechanics. We have faced the study of this issue and given a Kochen-Specker type theorem for the enriched lattice (Domenech et al., 2006). In this paper, we

give a topological version of that theorem, i.e. we study contextuality in terms of sheaves.

\section{Basic Notions}

We recall from (Goldblatt, 1986; Mac Lane and Moerdijk, 1992) and (Maeda and Maeda, 1970) some notions of sheaves and lattice theory that will play an important role in what follows. First, let $(A, \leq)$ be a poset and $X \subseteq A$. $X$ is a decreasing set iff for all $x \in X$, if $a \leq x$ then $a \in X$. For each $a \in A$ we define the principal decreasing set associated to $a$ as $(a]=\{x \in A: x \leq a\}$. The set of all decreasing sets in $A$ is denoted by $A^{+}$, and it is well known that $\left(A^{+}, \subseteq\right)$ is a complete lattice, thus $\left\langle A, A^{+}\right\rangle$is a topological space. We observe that if $G \in A^{+}$and $a \in G$ then $(a] \subseteq G$. Therefore $B=\{(a]: a \in A\}$ is a base of the topology $A^{+}$which we will refer to as the canonical base. Let $I$ be a topological space. A sheaf over $I$ is a pair $(A, p)$ where $A$ is a 
topological space and $p: A \rightarrow I$ is a local homeomorphism. This means that each $a \in A$ has an open set $G_{a}$ in $A$ that is mapped homeomorphically by $p$ onto $p\left(G_{a}\right)=\left\{p(x): x \in G_{a}\right\}$, and the latter is open in $I$. It is clear that $p$ is a continuous and open map. Local sections of the sheaf $p$ are continuous maps $\nu: U \rightarrow I$ defined over open proper subsets $U$ of $I$ such that $p \nu=1_{U}$. In particular we use the term global section only when $U=I$.

In a Boolean algebra $A$, congruences are identifiable to certain subsets called filters. $F \subseteq A$ is a filter iff it satisfies: if $a \in F$ and $a \leq x$ then $x \in F$ and if $a, b \in F$ then $a \wedge b \in F . F$ is a proper filter iff $F \neq A$ or, equivalently $0 \notin F$. If $X \subseteq A$, the filter $F_{X}$ generated by $X$ is the minimum filter containing $X$. A proper filter $F$ is maximal iff the quotient algebra $A / F$ is isomorphic to $\mathbf{2}$, being $\mathbf{2}$ the two elements Boolean algebra. It is well known that each proper filter can be extended to a maximal one.

We denote by $\mathcal{O} \mathcal{M L}$ the variety of orthomodular lattices. Let $L=$ $\langle L, \vee, \wedge, \neg, 0,1\rangle$ be an orthomodular lattice. Given $a, b, c$ in $L$, we write: $(a, b, c) D$ iff $(a \vee b) \wedge c=(a \wedge c) \vee(b \wedge c) ;(a, b, c) D^{*}$ iff $(a \wedge b) \vee c=(a \vee c) \wedge(b \vee c)$ and $(a, b, c) T$ iff $(a, b, c) D,(\mathrm{a}, \mathrm{b}, \mathrm{c}) D^{*}$ hold for all permutations of $a, b, c$. An element $z$ of a lattice $L$ is called central iff for all elements $a, b \in L$ we have $(a, b, z) T$. We denote by $Z(L)$ the set of all central elements of $L$ and it is called the center of $L . Z(L)$ is a Boolean sublattice of $L$ (Maeda and Maeda, 1970; Theorem 4.15).

\section{Sheaf-theoretic view of contextuality}

Let $\mathcal{H}$ be the Hilbert space associated to the physical system and $L(\mathcal{H})$ be the set of closed subspaces on $\mathcal{H}$. If we consider the set of these subspaces ordered by inclusion, then $L(\mathcal{H})$ is a complete orthomodular lattice (Maeda and Maeda, 1970). It is well known that each self-adjoint operator $\mathbf{A}$ that represents a physical magnitude $A$ may be associated with a Boolean sublattice $W_{A}$ of $L(\mathcal{H})$. More precisely, $W_{A}$ is the Boolean algebra of projectors $\mathbf{P}_{i}$ of the spectral decomposition $\mathbf{A}=\sum_{i} a_{i} \mathbf{P}_{i}$. We will refer to $W_{A}$ as the spectral algebra of the operator $\mathbf{A}$. Any proposition about the system is represented by an element of $L(\mathcal{H})$ which is the algebra of quantum logic introduced by G. Birkhoff and J. von Neumann (Birkhoff and von Neumann, 1936).

Assigning values to a physical quantity $A$ is equivalent to establishing a Boolean homomorphism $v: W_{A} \rightarrow \mathbf{2}$ (Isham, 1998). Thus, it is natural to consider the following definition which provides us with a compatibility 
condition:

Definition 3.1 Let $\left(W_{i}\right)_{i \in I}$ be the family of Boolean sublattices of $L(\mathcal{H})$. A global valuation over $L(\mathcal{H})$ is a family of Boolean homomorphisms $\left(v_{i}\right.$ : $\left.W_{i} \rightarrow \mathbf{2}\right)_{i \in I}$ such that $v_{i}\left|W_{i} \cap W_{j}=v_{j}\right| W_{i} \cap W_{j}$ for each $i, j \in I$.

Kochen-Specker theorem (KS) precludes the possibility of assigning definite properties to the physical system in a non-contextual fashion (Kochen and Specker, 1967). An algebraic version of KS theorem is given by (Domenech and Freytes, 2005; Theorem 3.2):

Theorem 3.2 If $\mathcal{H}$ be a Hilbert space such that $\operatorname{dim}(\mathcal{H})>2$, then a global valuation over $L(\mathcal{H})$ is not possible.

It is also possible to give a topological version of this theorem in the frame of local sections of sheaves. In fact, let $L$ be an orthomodular lattice. We consider the family $\mathcal{W}_{L}$ of all Boolean subalgebras of $L$ ordered by inclusion and the topological space $\left\langle\mathcal{W}_{L}, \mathcal{W}_{L}^{+}\right\rangle$. On the set

$$
E_{L}=\{(W, f): W \in \mathcal{W}, f: W \rightarrow \mathbf{2} f \text { is a Boolean homomorphism }\}
$$

we define a partial ordering given by $\left(W_{1}, f_{1}\right) \leq\left(W_{2}, f_{2}\right)$ iff $W_{1} \subseteq W_{2}$ and $f_{1}=f_{2} \mid W_{1}$. Thus we can consider the topological space $\left\langle E_{L}, E_{L}^{+}\right\rangle$whose canonical base is given by the principal decreasing sets $((W, f)]=\{(G, f \mid$ $G): G \subseteq W\}$. By simplicity $((W, f)]$ is noted as $(W, f]$.

Definition 3.3 The map $p_{L}: E_{L} \rightarrow \mathcal{W}_{L}$ such that $(W, f) \mapsto W$ is a sheaf over $\mathcal{W}_{L}$ called spectral sheaf associated to the orthomodular lattice $L$.

Let $\nu: U \rightarrow E_{L}$ be a local section of $p_{L}$. By (Domenech and Freytes, 2005; Proposition 4.2), for each $W \in U$ we have that $\nu(W)=(W, f)$ for some Boolean homomorphism $f: W \rightarrow \mathbf{2}$ and if $W_{0} \subseteq W$, then $\nu\left(W_{0}\right)=\left(W_{0}, f \mid\right.$ $\left.W_{0}\right)$. From a physical perspective, we may say that the spectral sheaf takes into account the whole set of possible ways of assigning truth values to the propositions associated with the projectors of the spectral decomposition $\mathbf{A}=\sum_{i} a_{i} \mathbf{P}_{i}$. The continuity of a local section of $p$ guarantees that the truth value of a proposition is maintained when considering the inclusion of subalgebras. In this way, the compatibility condition 3.1 of the Boolean valuation with respect to the intersection of pairs of Boolean sublattices of 
$L(\mathcal{H})$ is maintained. Thus, continuous local sections of $p_{L}$ are identifiable to compatible contextual valuations.

We use $\nu(a)=1$ to note that there exists $W \in U$ such that $a \in W$, $\nu(W)=(W, f]$ and $f(a)=1$. On the other hand, if $f: W \rightarrow 2$ is a Boolean homomorphism, $\nu:(W] \rightarrow E_{L}$ is such that for each $W_{i} \in(W]$, $\nu\left(W_{i}\right)=\left(W_{i}, f / W_{i}\right)$ is a local section of $p_{L}$. We call this a principal local section.

A global section $\tau: \mathcal{W}_{L} \rightarrow E_{L}$ of $p_{L}$ is interpreted as follows: the map assigns to every $W \in \mathcal{W}_{L}$ a fixed Boolean valuation $\tau_{w}: W \rightarrow \mathbf{2}$ obviously satisfying the compatibility condition. Thus, KS theorem in terms of the spectral sheaf reads (Domenech and Freytes, 2005; Theorem 4.3):

Theorem 3.4 If $\mathcal{H}$ is a Hilbert space such that $\operatorname{dim}(\mathcal{H})>2$ then the spectral sheaf $p_{L(\mathcal{H})}$ has no global sections.

\section{An algebraic study of modality}

With these tools, we are now able to build up a framework to include modal propositions in the same structure as actual ones. To do so we enrich the orthomodular lattice with a modal operator taking into account the following considerations: 1) Propositions about the properties of the physical system are interpreted in the orthomodular lattice of closed subspaces of the Hilbert space of the (pure) states of the system. 2) Given a proposition about the system, it is possible to define a context from which one can predicate with certainty about it together with a set of propositions that are compatible with it and, at the same time, predicate probabilities about the other ones. In other words, one may predicate truth or falsity of all possibilities at the same time, i.e. possibilities allow an interpretation in a Boolean algebra. In rigorous terms, for each proposition $P$, if we refer with $\diamond P$ to the possibility of $P$, then $\diamond P$ will be a central element of the orthomodular structure. $\quad 3$ ) If $P$ is a proposition about the system and $P$ occurs, then it is trivially possible that $P$ occurs. This is expressed as $P \leq \diamond P$. 4) Assuming an actual property and a complete set of properties that are compatible with it determines a context in which the classical discourse holds. Classical consequences that are compatible with it, for example probability assignments to the actuality of other propositions, shear the classical frame. These consequences are the same ones as those which would be obtained by considering the original actual property as a possible one. This is interpreted in the following way: if $P$ is a property of the system, $\diamond P$ is the smallest 
central element greater than $P$. From consideration 1) it follows that the original orthomodular structure is maintained. The other considerations are satisfied if we consider a modal operator $\diamond$ over an orthomodular lattice $L$ defined as $\diamond a=\operatorname{Min}\{z \in Z(L): a \leq z\}$ with $Z(L)$ the center of $L$.

Let $A$ be an orthomodular lattice. We say that $A$ is Boolean saturated if and only if for all $a \in A$ the set $\{z \in Z(A): z \leq a\}$ has a maximum (Domenech and Freytes, 2005). In this case, the maximum is denoted by $\square(a)$. In view of (Maeda and Maeda, 1970; Lemma 29.16), complete orthomodular lattices with an operator $e(a)=\bigvee\{z \in Z(L): z \leq a\}$, are examples of Boolean saturated orthomodular lattices. They form a variety of algebras $\langle A, \wedge, \vee, \neg, \square, 0,1\rangle$ of type $\langle 2,2,1,1,0,0\rangle$, noted as $\mathcal{O} \mathcal{M L}^{\square}$ (Domenech et al., 2006). $\mathcal{O} \mathcal{M L}^{\square}$ are axiomatized as follows:

$$
\begin{aligned}
& \text { S1 Axioms of } \mathcal{O M L} \quad \text { S5 } \quad \square(x \wedge y)=\square(x) \wedge \square(y) \\
& \text { S2 } \square x \leq x \quad \text { S6 } y=(y \wedge \square x) \vee(y \wedge \neg \square x) \\
& \text { S3 } \square 1=1 \quad \text { S7 } \quad \square(x \vee \square y)=\square x \vee \square y \\
& \mathrm{~S} 4 \quad \square \square x=\square x \quad \text { S8 } \quad \square(\neg x \vee(y \wedge x)) \leq \neg \square x \vee \square y
\end{aligned}
$$

On each algebra of $\mathcal{O} \mathcal{M L}^{\square}$ we can define the possibility operator as the unary operation $\diamond$ given by $\diamond x=\neg \square \neg x$. It satisfies $a \leq \diamond a$ and $\diamond a=\operatorname{Min}\{z \in Z(A): a \leq z\}$. If $L$ is an orthomodular lattice then there exists an orthomodular monomorphism $f: L \rightarrow A$ such that $A \in \mathcal{O} \mathcal{M} \mathcal{L}^{\square}$ (Domenech et al., 2006; Theorem 10). We refer to $A$ as a modal extension of $L$. In this case we can see the lattice $L$ as a subset of $A$. If $L^{\square} \in \mathcal{O} \mathcal{M} \mathcal{L}^{\square}$ is a modal extension of $L$, we define the possibility space of $L$ in $L^{\square}$ as $\diamond L=$ $\langle\{\diamond p: p \in L\}\rangle_{L^{\square}}$. If $W$ is a Boolean sublattice of $L$ then $\langle W \cup \diamond L\rangle_{L^{\square}}$ is a Boolean sublattice of $L^{\square}$; in particular $\diamond L$ is a Boolean sublattice of $Z\left(L^{\square}\right)$ (Domenech et al., 2006; Theorem 14). The possibility space represents the modal content added to the discourse about properties of the system.

\section{$5 \quad$ Sheaves and modality}

Let us consider $L^{\square}$ a modal extension of $L$. Then, the spectral sheaf $p_{L}$ is a subsheaf of $p_{L^{\square}}$. In this case we refer to $p_{L^{\square}}$ as a modal extension of $p_{L}$. It is clear that local sections of $p_{L}$ can be seen as local sections of $p_{L^{\square}}$. We define the set

$$
\operatorname{Sec}(\diamond L)=\left\{\nu:(\diamond L] \rightarrow E_{L^{\square}}: \nu \text { is principal section of } p_{L^{\square}}\right\}
$$


Since $\diamond L$ is a Boolean algebra, it is a subdirect product of $\mathbf{2}$. Thus, it always exists a Boolean homomorphism $f: \diamond L \rightarrow 2$, resulting $\operatorname{Sec}(\diamond L) \neq \emptyset$. From a physical point of view, $\operatorname{Sec}(\diamond L)$ represents all physical properties as possible properties. The fact that $\operatorname{Sec}(\diamond L) \neq \emptyset$ shows that, in the frame of possibility, one may talk simultaneously about all physical properties.

In the orthomodular lattice of the properties of the system, it is always possible to choose a context in which any possible property pertaining to this context can be considered as an actual one. We formalize this fact in the following definition and then we prove that this is always possible in our modal structure:

Definition 5.1 Let $L$ be an orthomodular lattice, $W$ a Boolean sublattice of $L, q \in W$ and $L^{\square}$ be a modal extension of $L$. If $\nu \in \operatorname{Sec}(\diamond L)$ such that $\nu(\diamond q)=1$ then an actualization of $q$ compatible with $\nu$ is an extension $\nu^{\prime}: U \rightarrow E_{L^{\square}}$ such that $\left(\langle W \cup \diamond L\rangle_{L^{\square}}\right] \in U$

Theorem 5.2 Let $L$ be an orthomodular lattice, $W$ a Boolean sublattice of $L, q \in W$ and $L^{\square}$ be a modal extension of $L$. If $\nu \in \operatorname{Sec}(\diamond L)$ such that $\nu(\diamond q)=1$ then there exists an actualization of $q$ compatible with $\nu$.

Proof: $\quad$ Suppose that $\nu(\diamond L)=(\diamond L, f)$. Let $F$ be the filter associated

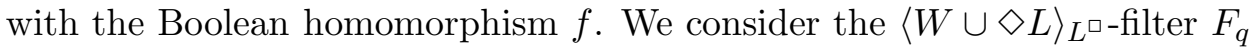
generated by $F \cup\{q\}$. $F_{q}$ is a proper filter. In fact: if $F_{q}$ is not proper, then there exists $a \in F$ such that $a \wedge q \leq 0$. Thus $q \leq \neg a$ being $\neg a$ a central element. But $\diamond q$ is the smallest Boolean element greater than $q$. Then $\diamond q \leq$ $\neg a$ or equivalently $\diamond q \wedge a=0$. And this is a contradiction since $\diamond q, a \in F$. Thus, we may extend $F_{q}$ to be a maximal filter $F_{M}$ in $\langle W \cup \diamond L\rangle_{L^{\square}}$. If we consider the natural projection $f_{F_{M}}:\langle W \cup \diamond L\rangle_{L^{\square}} \rightarrow\langle W \cup \diamond L\rangle_{L^{\square}} / F_{M} \approx \mathbf{2}$, the local section $\nu^{\prime}:\left(\langle W \cup \diamond L\rangle_{L^{\square}}\right] \rightarrow E_{L^{\square}}$ is an actualization of $q$ compatible with $\nu$.

The next theorem allows a representation of the Born rule in terms of continuous local sections of sheaves. This rule quantifies possibilities from a chosen spectral algebra.

Theorem 5.3 Let $L$ be an orthomodular lattice, $W$ a Boolean sublattice of $L$, and $\nu:(W] \rightarrow E_{L}$ a principal local section. If we consider a modal extension $L^{\square}$ of $L$ then there exists an extension $\nu^{\prime}: U \rightarrow E_{L^{\square}}$ such that $\langle W \cup \diamond L\rangle_{L^{\square}} \in U$. 
Proof: $\quad$ Suppose that $\nu(W)=(W, f)$. Let $i: W \rightarrow\langle W \cup \diamond L\rangle_{L^{\square}}$ be the Boolean canonical embedding. We see that there exists a Boolean homomorphism $f^{\prime}:\langle W \cup \diamond L\rangle_{L^{\square}} \rightarrow \mathbf{2}$ such that $f=f^{\prime} i=f^{\prime} \mid W$ since $\mathbf{2}$ is injective in the variety of Boolean algebras (Sikorski, 1948). Thus $\nu^{\prime}:\left(\langle W \cup \diamond L\rangle_{L^{\square}}\right] \rightarrow E_{L^{\square}}$ is the extension required.

We note that this reading of the Born rule is a kind of the converse of the possibility of actualizing properties given by Theorem 5.2.

Definition 5.4 Let $L$ an orthomodular lattice, $L^{\square}$ be a modal extension and $\nu \in \operatorname{Sec}(\diamond L)$. An actualization compatible with $\nu$ is a global section $\tau: \mathcal{W}_{L} \rightarrow E_{L}$ of $p_{L}$ such that $\tau(W \cap \diamond L)=\nu(W \cap \diamond L)$.

Theorem 5.5 Let $L$ be an orthomodular lattice. Then $p_{L}$ is a global section $\tau$ iff for each modal extension $L^{\square}$ there exists $\nu \in \operatorname{Sec}(\diamond L)$ such that $\tau$ is a compatible actualization of $\nu$.

Proof: $\quad$ Suppose that $p_{L}$ admits a global section $\tau: \mathcal{W}_{L} \rightarrow E_{L}$ and let $\tau(W)=\left(W, f_{W}\right)$. We consider the family $\left(A_{W}=W \cap \square L\right)_{W \in \mathcal{W}_{L}}$. Let $f_{0}: \bigcup_{W} A_{W} \rightarrow \mathbf{2}$ such that $f_{0}(x)=f_{W}(x)$ if $x \in W$. $f_{0}$ is well defined since $\tau$ is a global section. If we consider $\left\langle\bigcup_{W} A_{W}\right\rangle_{L^{\square}}$ the subalgebra of $L^{\square}$ generated by the join of the family $\left(A_{W}\right)_{W}$, it may be proved that it is a Boolean subalgebra of $\diamond L$. We can extend $f_{0}$ to a Boolean homomorphism $f_{0}^{\prime}:\left\langle\bigcup_{W} A_{W}\right\rangle_{L^{\square}} \rightarrow \mathbf{2}$. Since $\mathbf{2}$ is injective in the variety of Boolean algebras (Sikorski, 1948), then there exists a Boolean homomorphism $f: \diamond L \rightarrow \mathbf{2}$ which extends it to $f_{0}^{\prime}$. If we consider $\nu \in \operatorname{Sec}(\diamond L)$ such that $\nu(G)=(G, f \mid G)$, it result that $\tau$ is a compatible actualization of $\nu$. The converse is immediate.

To conclude we may say that the addition of modalities to the discourse about the properties of a quantum system enlarges its expressive power. At first sight it may be thought that this could help to circumvent contextuality, allowing to refer to physical properties belonging to the system in an objective way that resembles the classical picture. In view of the last theorem, since any global section of the spectral sheaf is a compatible actualization of a local one belonging to $\operatorname{Sec}(\diamond L)$, a global actualization that would correspond to a family of compatible valuations is prohibited. Thus, the theorem states that the contextual character of quantum mechanics is maintained even when the discourse is enriched with modalities. 
Acknowledgements This work was partially supported by the following grants: PICT 04-17687 (ANPCyT), PIP N ${ }^{o}$ 6461/05 (CONICET), UBACyT $\mathrm{N}^{o} \mathrm{X} 081$ and X204.

\section{References}

[1] Birkhoff, G. and von Neuman, J. (1936). The logic of quantum mechanics. Annals of Mathematics 27, 823-843.

[2] Dalla Chiara, M. L. (1981). Some metalogical pathologies of Quantum Logic. In: Beltrametti, E. and van Fraassen, B. (Eds.), Current Issues in Quantul Logic, Plenum Press, New York, 147-159 .

[3] Dalla Chiara, M. L., Giuntini, R. and Greechie, R. (2004). Reasoning in Quantum Theory. Sharp and Unsharp Quantum Logic, Kluwer Academic Publishers, Dordrecht.

[4] Dieks, D. (1988). The formalism of quantum theory: an objective description of reality. Annalen der Physik 7, 174-190.

[5] Dieks, D. (1989). Quantum mechanics without the projection postulate and its realistic interpretation. Foundations of Physics 19, 1397-1423.

[6] Dieks, D. (2005). Quantum Mechanics: an Intelligible Description of Reality?. Foundations of Physics 35, 399-415.

[7] Domenech, G. and Freytes, H. (2005). Contextual logic for quantum systems. Journal of Mathematical Physics 46, 012102-1 - 012102-9.

[8] Domenech, G., Freytes, H. and de Ronde, C. (2006). Scopes and limits of modality in quantum mechanics. Annalen der Physik 15, in press.

[9] van Fraassen, B. C. (1981). A modal interpretation of quantum mechanics. In: Beltrametti E. G. and van Fraassen, B. C. (Eds), Current issues in quantum logic, Plenum Press, New York, 229-258.

[10] van Fraassen, B. C. (1991). Quantum Mechanics: An Empiricist View, Clarendon, Oxford.

[11] Goldblatt, R. (1984). Orthomodularity is not elementary. The Journal of Simbolic Logic 49, 401-404.

[12] Goldblatt, R. (1986). Topoi: The categorical analysis of logic, Elsevier Science, Amsterdan. 
[13] Isham, C. and Butterfield, J. (1998). A topos perspective on the KochenSpecker theorem: I. International Journal of Theoretical Physics 37, 26692773 .

[14] Jauch, J. M. (1966). Foundations of Quantum Mechanics, Addison-Wesley Pub. Co., Massachusetts.

[15] Kochen, S. and Specker, E. P. (1967). The problem of hidden variables in quantum mechanics. Journal of Mathematics and Mechechanics 17, 9-87.

[16] Maeda, F. and Maeda, S. (1970). Theory of Symmetric Lattices, SpringerVerlag, Berlin.

[17] Mac Lane, S. and Moerdijk, I. (1992). Sheaves in Geometry and Logic: A first introduction to topos theory, Springer, Berlin.

[18] Sikorski, R. (1948). A theorem on extensions of homomorphism. Ann. Soc. Pol. Math. 21, 332-335. 